Experimental Induction of Coupling in Xenopus laevis, with the Production of Fertilised Eggs

IN the course of an investigation into the sexual cycle of Xenopus laevis, the South African clawed toad (Shapiro and Zwarenstein ${ }^{1}$, Shapiro and Shapiro ${ }^{2}$ ), certain outstanding differences in the sexual behaviour of this amphibian were observed under laboratory conditions as compared with natural conditions prevailing in the pond. It was noticed, among other things, that mating activity was never in any circumstances exhibited in captivity in the laboratory even at the height of the breeding season (July to September in South Africa).

In order to investigate the factors concerned with the mating reflex, toads fresh from the pond were brought into the laboratory at monthly intervals from August 1934. These toads were divided into three groups with appropriate controls. (Details of these experiments will be given in a forthcoming publication.)

In the first group, both the male and the female toads were injected with an acid extract of sheep's anterior pituitary, with an extract of pregnancy urine (prepared according to the method of Katzman and Doisy ${ }^{3}$ ) and a similarly prepared extract of male urine. In the second group, females only and not males were injected with these extracts. In the third group, males only and not females were injected similarly.

The results were as follows :-

A maximum percentage of coupling took place when both males and females were injected with either pregnancy urine or anterior pituitary extract.

A smaller percentage of coupling occurred when females only and not males were injected. This percentage rapidly dwindled to zero, the further from the breeding season in point of time the injections were made.

No coupling was observed when males only and not females were injected, even at the height of the breeding season.

In no case did the male urine extract induce the act of coupling or ovulation.

Colour change in Xenopus (which involves a pituitary mechanism, Hogben and Slome ${ }^{4}$ ) did not affect the mating reflex.

Several pairs of coupling toads were killed at the beginning of the act of mating and their oviducts were found to be entirely free of ova. Ovulation has also been observed without concomitant coupling.

In all, the mating reflex has been induced experimentally in 71 different pairs of toads with pregnancy urine extract and in 20 different pairs with anterior pituitary extract. The act of coupling lasts 24-48 hours, and fertilised ova were recovered from the containers of such coupling pairs. The ova were successfully reared for 4-5 months to the tadpole stage.

By injection of the above extracts coupling can be induced in the laboratory not only near the breeding season, but also in midsummer, that is, the midpoint between two breeding seasons. In all cases tadpoles have so far been reared successfully from such fertilised eggs.

The experiments also suggest :

(I) That a female stimulus initiates the response by activating the male.

(2) That the anterior pituitary is involved in this complex pattern of motor behaviour.
(3) That the pituitary mechanism associated with colour change in Xenopus laevis is not concerned with the mating reflex.

(4) That if the same chemical substance initiates ovulation as well as coupling, it initiates these two activities at different thresholds.

A further investigation into the details of the mating reflex is being carried out.

Department of Physiology,

University of Cape Town. Jan. 31 .

${ }^{1}$ Shapiro and Zwarenstein, J. Exp. Biol., 10, 4, 372; 1933.

Shapiro and Shapiro, J. Exp. Biol., 11, 1, 73; 1934 .

Hogben and Slome, Proc. Roy. Soc., B, 108, 10; 1931.

\section{Use of Cellulose Films in Palæontology}

REFERENCE has been made by $\mathrm{S}$. Leclerq ${ }^{1}$ and J. E. Appel' to the use of cellulose films or 'peels' in palæontology, but there does not seem to be any published account of the procedure involved. In the method outlined below, which was suggested to me by Prof. L. J. Wills, and used in the first place to record the internal structure of rugose corals, a cellulose cast is made from an etched surface, and structural detail stands out solely by reason of the optical effect of textural variation.

The cellulose solution is most easily prepared by diluting the commercial preparation 'Durofix' with about twice its volume of butyl acetate; I have also found very satisfactory the solution advocated by R. Graham ${ }^{3}$.

The cut surface of the coral is ground with fine carborundum, etched for five to ten seconds with dilute (10 per cent) hydrochloric acid, washed and allowed to dry; the finer the organic structure, the shorter is the time of etching required. The specimen is mounted on plasticine so that the etched surface is horizontal, and after moistening the latter with butyl acetate as a precaution against air bubbles, the cellulose solution is poured on to form a layer about $2 \mathrm{~mm}$. thick. The solution dries in 3-5 hours, and the edge of the film is then lifted from the specimen with a sharp blade, and the whole film peeled off. The film can conveniently be preserved between glass plates bound together at the edges.

The method is applicable to any fossil in which a difference in relief and texture between the organic parts and the surrounding matrix can be obtained by etching with hydrochloric or hydrofluoric acid. In the preparation of serial records it is advisable to embed the specimen in plaster of Paris before grinding; the distance between successive peels can be measured with a spherometer. Peels from corals and brachipods show not only the outlines of the skeletal elements, but also the detailed fibrous or lamellar structure of these; some which were prepared in 1931 are still free from contraction or discoloration.

An important possibility is the distribution of duplicate records of type specimens, since a great many peels can be made from a prepared surface without re-grinding or re-etching.

Department of Geology,

\section{A. J. Butler.}

Victoria University of Manchester. Feb. 28.

1 Annales de la Soc. Geol. Bela., 52, B24-27; 1928.

"Stain Technology", 1928, vol. 8, pp. 65-68. 